INPLASY

PROTOCOL

To cite: Sun et al. Metaanalysis of the effect of traditional Chinese exercise therapy on elderly women's balance ability and lower limb muscle strength. Inplasy protocol 202160114. doi: 10.37766/inplasy2021.6.0114

Received: 30 June 2021

Published: 30 June 2021

Corresponding author: Xing Wang

1305380127@qq.com

Support: 11DZ2261100.

Review Stage at time of this submission: Data analysis.

Conflicts of interest:

None declared.

\section{Meta-analysis of the effect of traditional Chinese exercise therapy on elderly women's balance ability and lower limb muscle strength}

\author{
Sun, S1; Zhao, Z2; Wang, W³.
}

Review question / Objective: To systematically evaluate the effect of traditional Chinese exercise therapy on the balance ability and lower limb muscle strength of elderly women.

Eligibility criteria: Research objects: According to the definition of the World Health Organization for the elderly, the elderly are required to be $\geq 60$ years old and female; the intervention method is traditional Chinese sports; the outcome indicators or part of the outcome indicators are balance ability and lower limb muscle strength.

INPLASY registration number: This protocol was registered with the International Platform of Registered Systematic Review and Meta-Analysis Protocols (INPLASY) on 30 June 2021 and was last updated on 30 June 2021 (registration number INPLASY202160114).

\section{INTRODUCTION}

Review question / Objective: To systematically evaluate the effect of traditional Chinese exercise therapy on the balance ability and lower limb muscle strength of elderly women.

Condition being studied: The effect of traditional Chinese exercise therapy on elderly women's balance ability and lower limb muscle strength. A total of 18 studies with a sample size of 908 were included. Meta analysis results show that traditional Chinese exercise therapy can significantly improve the static balance of elderly women.

\section{METHODS}

Participant or population: Elderly women 908.

Intervention: Traditional Chinese exercise therapy.

Comparator: Old women.

Study designs to be included: A computer search for randomized controlled trials of 
traditional Chinese exercise therapy in the databases of PubMed, The Cochrane Library, Web of Science, Embase, CNKI, CBM, Wanfang, and VIP.

Eligibility criteria: Research objects: According to the definition of the World Health Organization for the elderly, the elderly are required to be $\geq 60$ years old and female; the intervention method is traditional Chinese sports; the outcome indicators or part of the outcome indicators are balance ability and lower limb muscle strength.

Information sources: PubMed, The Cochrane Library, Web of Science, Embase, CNKI, CBM, Wanfang, and VIP.

Main outcome(s): A total of 18 studies with a sample size of 908 were included. Meta analysis results show that traditional Chinese exercise therapy can significantly improve the static balance of elderly women.

Quality assessment / Risk of bias analysis: Use the internationally recognized and widely used literature quality evaluation tool PEDro scale to evaluate the methodological quality of randomized controlled trials. This process was carried out by two researchers independently and reviewed each other. If there is a disagreement, the third author will discuss and decide whether to give a score.

Strategy of data synthesis: 8 Chinese and English databases including PubMed, The Cochrane Library, Web of Science, Embase, CNKI, CBM, Wanfang, and Weipu are searched by computer. The search period is from the beginning of the collection of each database to March 19, 2021. English search terms include Tai $\mathrm{Ji}$, taichi, health Qigong, Baduanjin, Taijiquan, Wuqinxi, Yijinjing, postural balance, balance, muscular strength, muscle strength, aged, elder, the elderly, old people, older adults, randomized controlled trial, randomized, trial, randomization. Chinese search terms include Taijiquan, Ba Duan Jin, Wu Qin Xi, Yi Jin Jing, Health Qigong, traditional exercises, randomized comparison, etc.
The search strategy adopts a combination of subject words and free words, and uses Boolean operations "AND" and or "OR" to combine (topic or title) connections, and is determined after repeated pre-checks, supplemented by manual search, if necessary Trace back the references of the included literature.

Subgroup analysis: Perform subgroup analysis according to test indicators, exercise type, exercise frequency, and exercise cycle.

Sensitivity analysis: In order to explore whether the heterogeneity between the studies is caused by a single study, the RevMan5.3 software was used for sensitivity analysis.

Country(ies) involved: China.

Keywords: traditional Chinese sports; elderly women; balance; lower limb muscle strength; meta-analysis.

Contributions of each author:

Author 1 - Wenxin Sun.

Author 2 - Qingying Zhao.

Author 3 - Xing Wang. 